\title{
Instalación ecoguiada del Balón de Contrapulsación Intraaórtico (BCPIA) vía arteria axilar por abordaje extratorácico en pacientes en espera de Trasplante Cardíaco
}

\author{
Oneglio Pedemonte ${ }^{1}$, Bernhard Westerberg ${ }^{2}$, Lorenzo Merello ${ }^{3}$, Cristopher Araya $^{4}$, Luis Becerra ${ }^{2}$, Abigail Peña $^{5}$, \\ Ernesto Aránguiz-Santander 6 .
1 Anestesiólogo Cardiovascular, Jefe Unidad Cuidados Intensivos Cardiovasculares, Hospital Dr. Gustavo Fricke, Viña del Mar, Chile. 2 Cardiólogo Hemodinamista, Unidad Cuidados Intensivos Cardiovasculares, Hospital Dr. Gustavo Fricke, Viña del Mar, Chile. 3 Anestesiólogo Cardiovascular, Hospital Dr. Gustavo Fricke, Viña del Mar, Chile. Docente cátedra Anestesiología y Reanimación Universidad de Valparaíso, Valparaíso, Chile. 4 Anestesiólogo, Hospital Dr. Gustavo Fricke, Viña del Mar, Chile. 5 Residente Cardiología Universidad de Valparaíso, Hospital Dr. Gustavo Fricke, Viña del Mar, Chile. 6 Cirujano Cardiovascular, Jefe Servicio de Cirugía Cardiovascular, Hospital Dr. Gustavo Fricke, Viña del Mar, Chile. \\ Financiamiento: Ninguno \\ Conflicto de interés: Ninguno
}

El balón de contrapulsación intraaórtico (BCPIA) es el dispositivo más frecuentemente utilizado para otorgar soporte mecánico en pacientes con insuficiencia cardíaca terminal y compromiso hemodinámico refractario al soporte inotrópico. Se han utilizado diferentes tipos de abordajes para la instalación del BCPIA a través de las extremidades superiores, ya sea vía arteria subclavia o últimamente vía arteria axilar.

Con el objetivo de evitar la inmovilidad física asociada al BCPIA instalado vía arteria femoral, aumentar la comodidad del paciente, simplificar la técnica de instalación del catéter y facilitar los cuidados de enfermería necesarios para su mantención, hemos diseñado y utilizado un abordaje simple y seguro para su inserción. Bajo técnica ecoguiada y utilizando un set de micro punción, se realizó la canulación de la arteria axilar en su porción externa (lateral al borde externo del músculo pectoral menor). Con la ayuda de fluoroscopía, el BCPIA fue avanzado hasta posicionarlo en la aorta descendente bajo la emergencia de la arteria subclavia izquierda.

En dos pacientes con insuficiencia cardíaca terminal INTERMACS 2, con deambulación restringida debido a la necesidad permanente de soporte inotrópico y BCPIA femoral, bajo visión ecoguiada se realizó la reinstalación de este catéter a través de la arteria axilar izquierda, utilizando anestesia local y fluoroscopía en el laboratorio de hemodinamia. La rehabilitación fue posible rápidamente en ambos pacientes, realizándose el transplante cardíaco ortotópico después de 10 y 69 días de soporte mecánico con BCPIA, sin mayores efectos adversos. 


\section{Echo -guided installation of Inta aortic balloon counterpulsation via the extra thoracic axillary artery}

Intra aortic balloon counterpulsation (IABP) is the most frequently used procedure to give mechanic support in patients with terminal heart failure and hemodynamic compromise refractory to inotropic support. Different approaches have been utilized to install upper extremity IABP, via either the axillary or subclavian arteries.

In order to circumvent the limitations associated to long lasting femoral IABP support, simplify the technique, increase patient comfort and facilitate nursing care, we designed a simplified approach. Using echo guidance, the axillary artery was accessed with a micropuncture set just outside the external margin of the pectoral minor muscle. With the help of fluoroscopy the IABP catheter was advanced and its correct position in the descending aorta was confirmed

Two patients in INTERMACS 2 end stage heart failure, bedridden due to permanent inotropic support and femoral IABP, underwent echo guided axillary IABP placement under local anesthesia and fluoroscopy in the catheterization laboratory. Both patients gained rapid access to rehabilitation and received orthotopic heart transplantation after 10 and 69 days of uneventful IABP support. 


\section{Introducción:}

Los pacientes con insuficiencia cardíaca terminal (ICT) frecuentemente sufren descompensaciones agudas mientras se encuentran en espera de transplante cardíaco (TC), requiriendo en ocasiones de hospitalización para manejo depletivo y soporte hemodinámico farmacológico $^{1}$. Un porcentaje de estos pacientes requieren de apoyo inotrópico permanente para lograr la estabilidad hemodinámica y perfusión orgánica adecuada. El Balón de Contrapulsación Intraaórtico (BCPIA) es al dispositivo más utilizado para asistencia mecánica en pacientes con ICT y compromiso hemodinámico refractario al soporte inotrópico ${ }^{2}$. Sin embargo, su uso implica la hospitalización e inmovilización prolongada de los pacientes.

La inserción del BCPIA se ha realizado clásicamente través de la arteria femoral común (AFC), un abordaje extratorácico que es una localización incómoda que limita la deambulación y entrenamiento de los pacientes. Ello tiene una repercusión negativa en la condición física, nutricional y mental de los pacientes, que se asocia a una mayor morbi-mortalidad y retraso en la recuperación postoperatoria.

Los abordajes transtorácicos evitan esta limitación mientras se conservan los beneficios hemodinámicos del BCPIA. Aunque resultaba cómodo para la deambulación, las primeras técnicas descritas eran complejas e involucraban la inserción quirúrgica del dispositivo directamente en la aorta torácica descendente ${ }^{3}$. De esta manera, las grandes arterias de las extremidades superiores surgieron como una alternativa menos invasiva para la inserción del BCPIA por abordaje transtorácico. La arteria axilar (AA) ha sido utilizada en los últimos años para la inserción de este dispositivo.

La AA, continuación de la arteria subclavia, se extiende desde el borde lateral de la primera costilla hasta el músculo teres mayor. En su trayecto se divide en una porción interna, media y externa según su posición sea medial, bajo o lateral al músculo pectoral menor respectivamente ${ }^{4}$ (Figura 1). Al ser un vaso extratorácico, de fácil acceso percutáneo y poseer abundante circulación colateral para la extremidad superior, la AA constituye una alternativa segura y menos invasiva para la inserción del BCPIA, conservando además la movilidad de los pacientes.

Las primeras técnicas descritas involucraban la exposición quirúrgica de la $\mathrm{AA}$, y posterior anastomosis a una prótesis tubulada ${ }^{5}$ o injerto venoso ${ }^{6}$, a través de los cuales se insertaba el dispositivo. La inserción percutá-

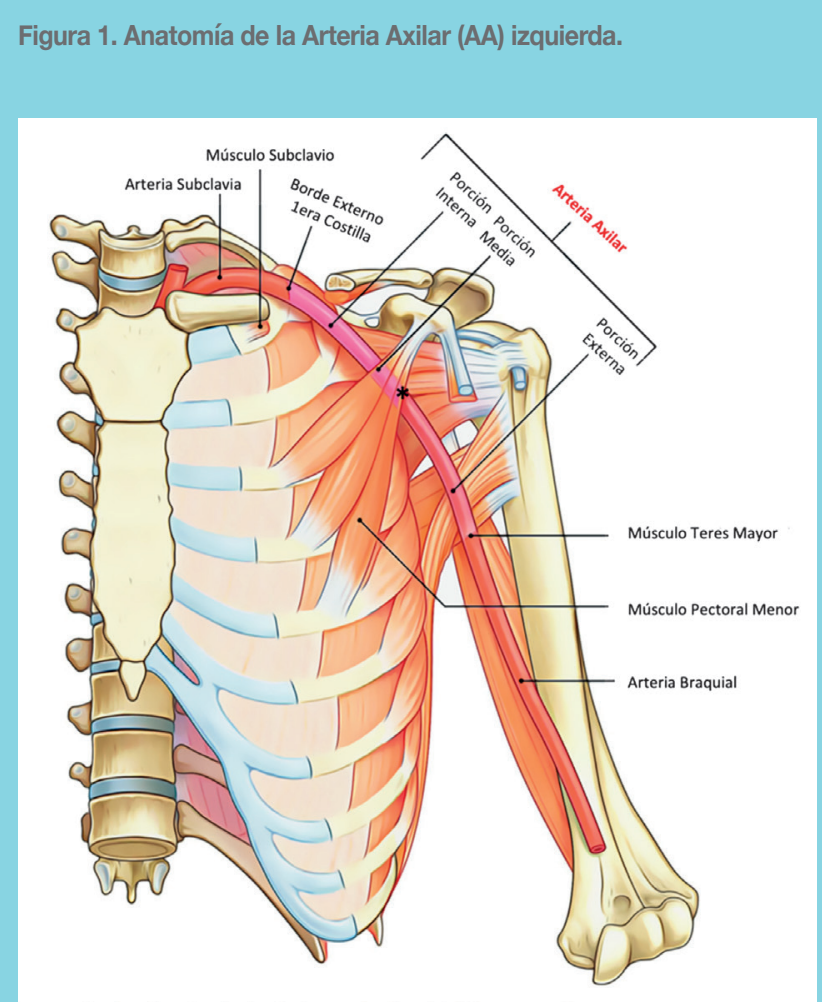

La AA es la continuación de la Arteria Subclavia, y comienza en el borde lateral de la primera costilla. Su trayecto se divide en 3 porciones según su posición respecto al músculo pectoral menor (PM). La porción interna se localiza desde el inicio de la AA hasta el borde interno del PM. La porción media se localiza bajo el PM, y emite ramos arteriales 4 que contribuyen a la circulación colateral de la extremidad superior. La porción externa se localiza entre el PM y el músculo teres mayor, lugar donde pasa a llamarse arteria braquial. El asterisco indica la zona ideal de punción.

nea fue descrita posteriormente; sin embargo, la técnica seguía siendo compleja. Con la finalidad de facilitar el entrenamiento pre trasplante cardíaco (TC) en pacientes con ICT y compromiso hemodinámico refractario, describimos a continuación una técnica ecoguiada y percutánea para la inserción del BCPIA a través de la porción externa de la AA.

\section{Presentación del caso}

Dos pacientes con ICT en lista de espera para TC fueron ingresados a la unidad de cuidados intensivos (UCI) cardiovasculares dado descompensación aguda de su cardiopatía de base. El compromiso hemodinámico y mala perfusión orgánica persistió pese a soporte ino- 
Figura 2. Punción ecoguiada de la AA.
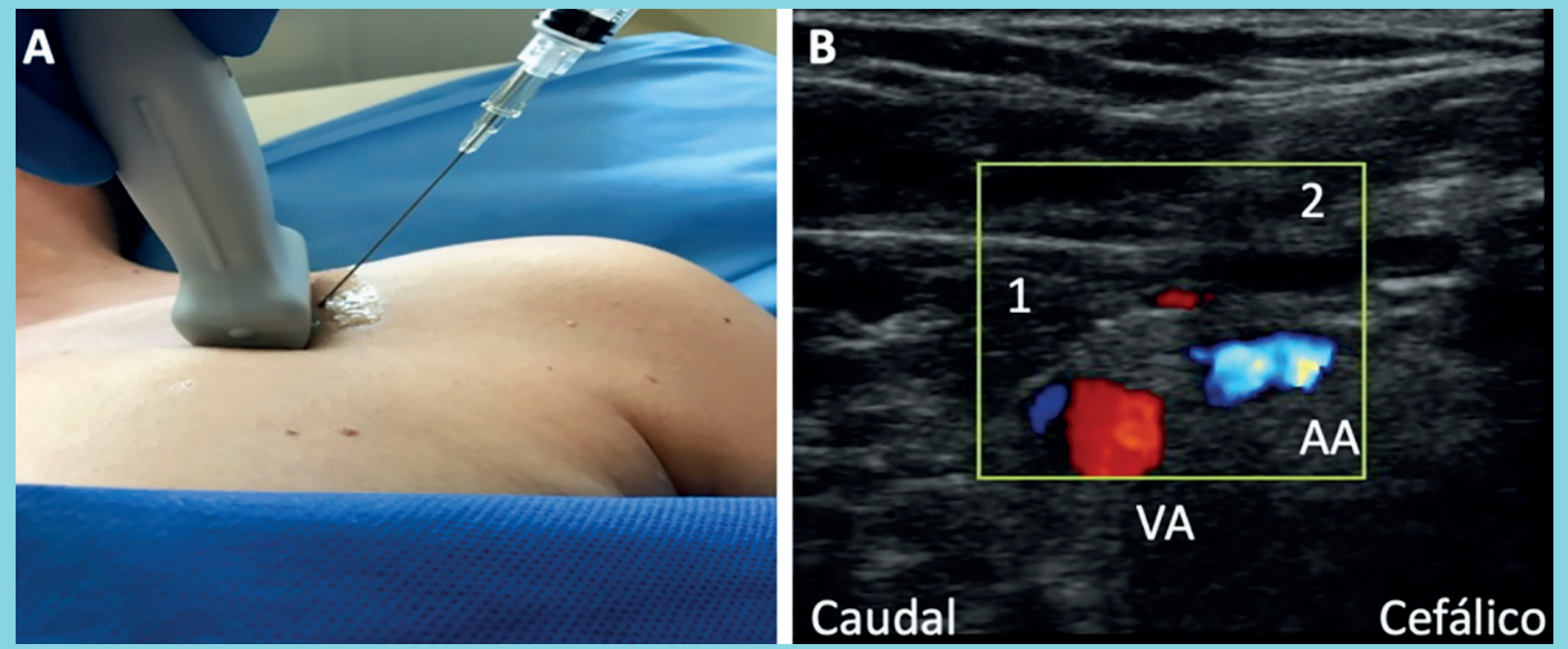

(A)El transductor ecográfico es posicionado perpendicular a la clavícula izquierda, justo a nivel del proceso coracoides. (B) Eje corto de la porción externa de los vasos axilares, a nivel del borde externo del músculo pectoral menor (1). La arteria axilar (AA) se encuentra en relación cefálica respecto a la vena (VA), y ambos vasos se encuentran cubiertos en esa zona solo por el músculo pectoral mayor (2). Esta ventana permite la punción de la AA por técnica fuera de plano.

trópico óptimo, por lo que se les instaló un BCPIA vía arteria femoral común (AFC). Debido a la incertidumbre respecto al tiempo de espera para el TC y con el objetivo de facilitar la rehabilitación de los pacientes, se consideró la instalación del BCPIA vía AA izquierda. El procedimiento fue realizado en pabellón de hemodinamia utilizando monitorización estándar. Bajo técni- ca estéril, y utilizando un transductor ecográfico linear 6-13 MHz (HFC38 Sonosite Edge II, Fujifilm Sonosite Inc. Bothell, WA, USA), se procedió a identificar el límite entre la porción media y externa de la AA izquierda. El transductor fue posicionado perpendicular a la clavícula izquierda a nivel del proceso coracoides (Figura 2A). Con sutiles cambios en la angulación del

Figura 3. Punción de arteria axilar (AA), inserción del introductor y guía del BCPIA en aorta descendente (AD) guiado por fluroscopia
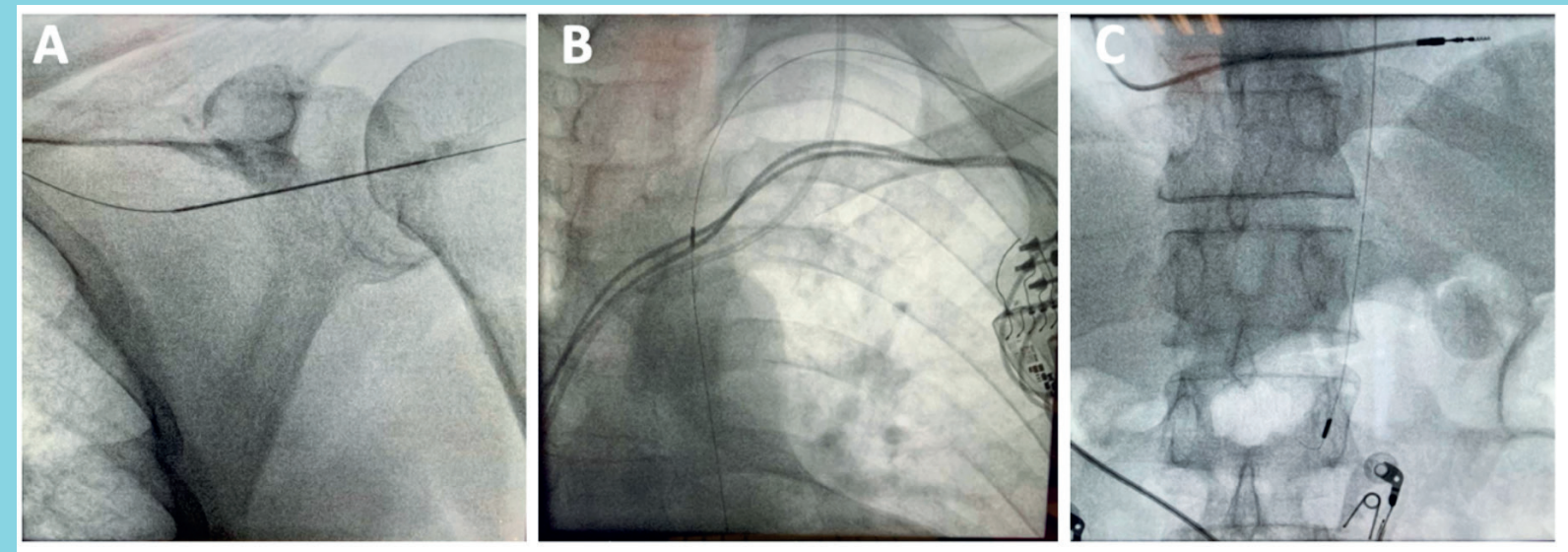

(A) Con set de micropunción y visión ecoguiada se realizó canulación de AA. La correcta posición de la guía metálica fue corroborada con fluoroscopia. (B) Una vez instalado el introductor del BCPIA, se insertó un catéter JR 45 French, a través del cual se maniobró y posicionó la guía del BCPIA. (C) Guía metálica correctamente posicionada en $A D$. 
transductor ("tilt"), se identificó la porción externa de la AA inmediatamente lateral al músculo pectoral menor y en una posición cefálica a la vena axilar, ambos vasos cubiertos solo por el músculo pectoral mayor (Figura 2B).

Tras infiltrar los planos subcutáneos con lidocaína 2\%, y utilizando una técnica fuera de plano, se puncionó la AA con una aguja 21G (Micropuncture vascular access kit, Cook Medical Bloomington Indiana, USA) en un ángulo de 45 grados respecto a la piel (Figura 2A). Mediante el uso de fluoroscopia, se posicionó una guía metálica 0.018" en el lumen arterial (Siemens, Axiom Artis, siemens Healthcare $\mathrm{GmbH}$ ). Con técnica de Seldinger, se instaló un introductor 4 French a través de la guía metálica, la que posteriormente se remplazó por una guía 0.035”x 2.60 (uniQual Asahi Intecc). A través de ésta, se realizó el cambio de introductor a uno de 8 Fr. Bajo fluoroscopia, la guía del nuevo BCPIA se posicionó en la aorta descendente torácica con ayuda de un catéter JR 4.0 de 5 French (Figura 3 A-C). A través de esta última guía, e inmediatamente después de retirar el BCPIA femoral, se instaló un nuevo catéter de $40 \mathrm{ml}$ (Maquet Getinge NJ USA). Una vez confirmado el correcto posicionamiento del BCPIA, se procedió a reiniciar la asistencia mecánica 1:1 sin dificultades (Figura 4).

Ambos pacientes tuvieron excelente tolerancia durante el procedimiento, y en 24 horas lograron la deambulación e inicio sin limitaciones de la rehabilitación. Un paciente fue transplantado tras 10 días de soporte mecánico mientras que el segundo fue asistido por 69 días. En ninguno de los dos casos ocurrió migración del dispositivo.

En ambos casos el destete del BCPIA se realizó 48 horas posteriores al TC y en uno de ellos el catéter fue retirado solo con compresión mecánica mientras que en el paciente que recibió soporte mas prolongado, el retiro se realizó utilizando un dispositivo de cierre percutáneo (Perclose ProGlide, Abbott Vascular Inc, USA). No se registraron complicaciones asociadas a la instalación, mantención y retiro del BCPIA.

\section{Discusión:}

El BCPIA ha demostrado beneficios como una terapia costo efectiva para otorgar soporte mecánico en pacientes con ICT y compromiso hemodinámico refractario a soporte inotrópico, funcionando en estos casos como puente al $\mathrm{TC}^{7,8}$.

Las arterias de las extremidades superiores se han uti-
Figura 4: Posición del BCPIA en aorta descendente (AD).

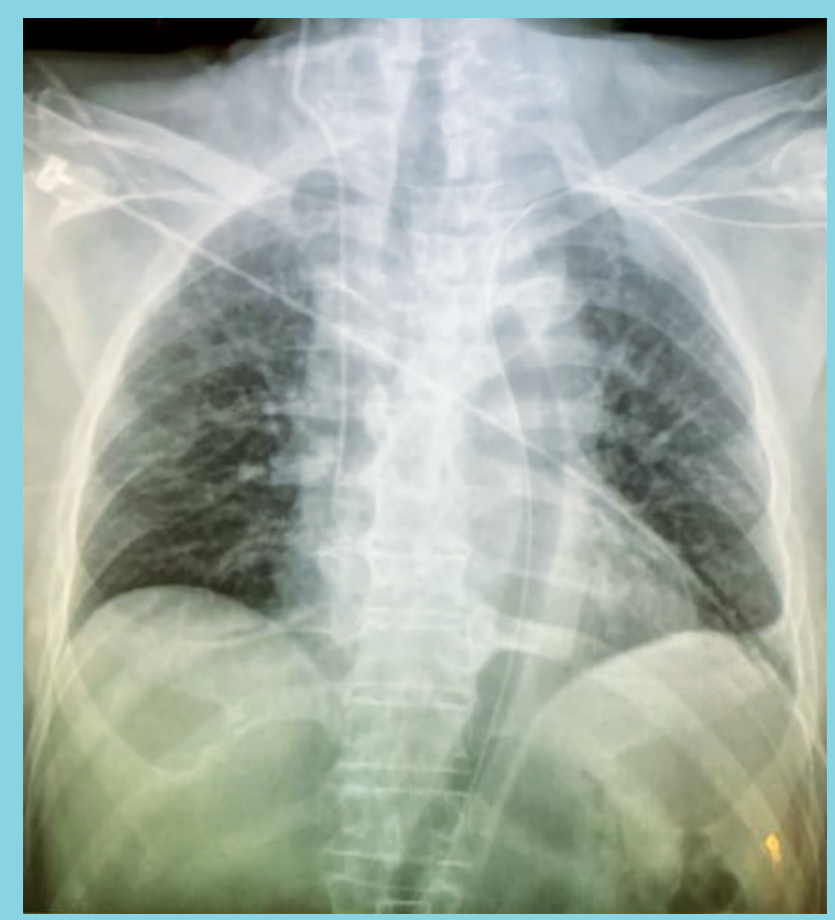

BCPIA instalado a través de la AA izquierda. Su correcto posicionamiento se confirmo con fluroscopia, logrando asistencia mecánica inmediata sin complicaciones.

lizado para la asistencia mecánica con BCPIA sin interumpir la movilidad de los pacientes. Sin embargo, su inserción a través de la arteria subclavia involucra alto riesgo de neumotórax. Por otro lado, con el uso de la arteria braquial se han reportado casos de isquemia de extremidad superior posterior a la inserción del dispositivo $^{9}$.

La AA es un acceso ideal, pues la inserción del dispositivo por este abordaje, además de permitir un soporte hemodinámico prolongado sin mayores complicaciones, facilita el desarrollo de un adecuado plan de rehabilitación. Lo anterior implica una mayor sobrevida de los pacientes en espera de TC y mejores resultados postoperatorios ${ }^{10,11}$.

Las primeras técnicas de abordaje de la AA fueron publicadas en el año 1978 y 2000 por Mayer ${ }^{5}$ y H'Doubler6', respectivamente. Sin embargo, su implementación era difícil dado que involucraban una intervención quirúrgica. En el año 2013, Estep et al. publicaron un método de inserción percutánea, en el cual se avanzaba una guía metálica en punta de J instalada a través de la arteria femoral o braquial hasta la AA para facilitar su loca- 
lización y punción bajo flouroscopía ${ }^{12}$. La instalación del BCPIA mediante este método permitió un soporte hemodinámico entre 3 a 152 días, siendo la disfunción y desplazamiento del dispositivo las complicaciones mas frecuentes. En nuestro reporte, en ambos pacientes se instaló un BCPIA vía AA utilizando una técnica percutánea simplificada y eco guiada, permitiendo en ambos casos una rápida deambulación y rehabilitación. El dispositivo permitió soporte mecánico durante 10 y 69 días, período durante el cual no se reportaron complicaciones.

De esta manera, la AA izquierda es una alternativa válida y segura para la inserción del BCPIA, pero debe ser abordada idealmente entre sus porciones media y lateral (en el borde lateral del músculo pectoral menor). Esto es clínicamente relevante debido a que reduce el riesgo de neumotórax, lesión del plexo braquial y hematoma en el sitio de punción ya que corresponde a una zona fácilmente compresible.

El uso de ultrasonografía permitió identificar y puncionar fácilmente la porción externa de la AA, evitando la exposición quirúrgica del vaso y la necesidad de otro acceso vascular para facilitar su punción por fluoroscopía.
Creemos que la inserción del BCPIA en la porción externa de la AA permite otorgar un soporte hemodinámico con la misma tasa de efectividad respecto a otras posiciones. Además, preserva la circulación colateral de la extremidad superior en caso de trombosis o reducción del flujo arterial por el introductor. Al ser un sitio de mayor comodidad para los pacientes, facilita su deambulación y rehabilitación. Además, este segmento arterial está en una zona fácilmente accesible tanto para la compresión directa como para la instalación de dispositivos de cierre percutáneo una vez retirado el BCPIA.

\section{Conclusión:}

Los pacientes con ICT pueden llegar a requerir soporte mecánico prolongado con BCPIA para mantener la estabilidad hemodinámica y perfusión orgánica. La inserción clásica de este dispositivo vía AFC implica inmovilización y deterioro funcional de los pacientes en lista de espera para TC. Las técnicas de inserción del BCPIA han evolucionado a lo largo del tiempo con la finalidad de encontrar una opción que evite esta limitación. La porción externa de la arteria axilar constituye una alternativa válida, segura y cómoda para la inserción de BCPIA. 


\section{Referencias:}

1 FRIEDRICH EB, BÖHM M. Management of end stage heart failure. Heart 2007; 93:626-631.

2 GONZÁLEZ LS, CHANEY MA. Intraaortic Balloon Pump Counterpulsation, Part I: History, Technical Aspects, Physiologic Effects, Contraindications, Medical Applications/Outcomes. Anesth Analg. 2020 Sep;131(3):776-791.

3 BURACK JH ET al. Transthoracic Intraaortic Balloon Pump: A Simplified Technique. Ann Thorac Surg 1996; 62:299-301.

4 SCHÄFER U, HO Y, FRERKER C, SCHEWEL D, SANCHEZ-QUINTANA D, SCHOFER J, BIJUKLIC K, MEINCKE F, THIELSEN T, KREIDEL F, KUCK KH. Direct percutaneous access technique for transaxillary transcatheter aortic valve implantation: "the Hamburg Sankt Georg approach". JACC Cardiovasc Interv. 2012 May;5(5):477-486.

5 MAYER JH. Subclavian artery approach for insertion of intra-aortic balloon. J Thorac Cardiovasc Surg. 1978; 76: 61-63.

6 H'DOUBLER PB, H'DOUBLER WZ, BIEN RC, JANSEN DA. A Novel Technique for Intraaortic Balloon Pump Placement via the Left Axillary Artery in Patients Awaiting Cardiac Transplantation. Cardiovascular Surgery. 2000;8(6):463-465.

7 GONZÁLEZ LS, CHANEY MA. Balloon Pump Counterpulsation Part II: Perioperative Hemodynamic Support and New Directions. Anesth Analg. 2020 Sep;131(3):792-807.

8 NWAEJIKEA N, SONB AY, MILANOA CA, DANESHMANDA MA. Is there a role for upper-extremity intra-aortic balloon counterpulsation as a bridge-to-recovery or a bridge-to-transplant in the treatment of end-stage heart failure? Interactive CardioVascular and Thoracic Surgery 25 (2017) $654-658$

9 NOËL BM, GLEETON O, BARBEAU GR. Transbrachial insertion of an intra-aortic balloon pump for complex coronary angioplasty. Catheter Cardiovasc Interv. 2003 Sep;60(1):369; discussion 40 .

10 HUCKABY LV, SEESE LM, MATHIER MA, HICKEY GW, KILIC A. Intra-Aortic Balloon Pump Bridging to Heart Transplantation: Impact of the 2018 Allocation Change. Circ Heart Fail. 2020 Aug;13(8):e006971.

11 ENGELMAN DT, BEN ALI W, WILLIAMS JB, PERRAULT LP, REDDY VS, ARORA RC, ROSELLI EE, KHOYNEZHAD A, GERDISCH M, LEVY JH, LOBDELL K, FLETCHER N, KIRSCH M, NELSON G, ENGELMAN RM, GREGORY AJ, BOYLE EM. Guidelines for Perioperative Care in Cardiac Surgery: Enhanced Recovery After Surgery Society Recommendations. JAMA Surg. 2019 Aug 1;154(8):755-766.

12 ESTEP JD, CORDERO-REYES AM, BHIMARAJ A, TRACHTENBERG B, KHALIL N, LOEBE M, BRUCKNER B, ORREGO CM, BISMUTH J, KLEIMAN NS, et al. Percutaneous placement of an intra-aortic balloon pump in the left axillary/subclavian position provides safe, ambulatory longterm support as bridge to heart transplantation. JACC Heart Fail. 2013; 1:382-388. 